\title{
Differences Among Visual, Auditory, and Kinesthetic Memorization Procedures when Children in Upper Elementary Schools are Memorizing a Text
}

\author{
Julie Beardslee ${ }^{1}$ and Patricia Talarczyk ${ }^{1}$ \\ ${ }^{1}$ Mentor High School, Mentor, OH, USA
}

\section{$\underline{\text { ABSTRACT }}$}

In education, a variety of methods for learning or memorizing content are often used, from reading a passage to listening to a lecture. This plethora of available strategies causes those involved in the educational field to wonder if there is a type of device that helps students best retain information. The study described in this paper determined whether children in the 4th and 5th grade memorize a visual text most effectively when given either a visual, auditory, or kinesthetic strategy. A total of 56 respondents were selected from a local elementary school and divided into three equal groups to memorize as much of a 151-word poem as possible. After students were given 15 minutes to utilize their given device, an assessment was given, during which they had to fill in 15 missing words from the poem they had been assigned. Based on scores from the assessment, this study was able to show that the visual device yielded a higher mean score of 8.5 than either the auditory score of 6.7 and a kinesthetic score of 6.5. A series of t-tests were conducted to conclude that the visual mean produced a p-value of 0.08 against audio and 0.09 against kinesthetic, which is significant at a level of 0.10 . This evidence suggests that when young children are assigned the task of memorizing a short passage, visual devices may be the most optimal choice.

\section{Introduction}

Since the era of Ancient Greece and Rome, many philosophers have greatly contributed to the development of memorization strategies. Alessandro Vatri (2015) analyzes numerous texts from Ancient Greece to compare some of the many memorization procedures that were commonly used during this period. These memorization procedures consist predominantly of mnemonic devices. One mnemonic device described in this article is the use of meter and rhythm as an aid, which is constructed around rhythmic patterns that set limits on the phonetic features of words, such as the number of syllables or the pronunciation. This mnemonic device is believed to be effective because setting these limits restricts the range of words that can be incorrectly inserted when recalling a given point of a sentence (Vatri, 2015). Vatri also describes using repetition, organization, and conciseness as mnemonic aids and uses the beliefs of numerous scholars from Ancient Greece to support the descriptions of these devices. The information in the article provided by Vatri is important when considering memorization procedures in the twenty-first century because the devices set a strong foundation for the mnemonic strategies that are used in modern times. Gopal P. Sarma (2015) furthers the notion of how memorization procedures have developed by comparing the devices used today with the strategies from Ancient Greece and Egypt. Before the 15,000-word Iliad or the 200,000-word Mahabharata were transcribed, poetic structure and repetition were the most common techniques utilized to memorize such large texts (Sarma, 2015). In contrast, Sarma found that mnemonic devices were the most common for memorizing modern memory tasks, including speed cards and historic dates (Sarma, 2015). Vatri's and Sarma's analytical research are significant when considering modern memorization procedures because their findings support how mnemonic devices originated and are still common in today's society, while also comparing different ancient strategies to mnemonic devices. 
Another key component when considering memorization procedures is brain plasticity. Brain plasticity, or neuroplasticity, is the idea that the brain is constantly changing and adapting to the world around it, as stated by E.H. Chudler (2019). Neuroplasticity typically occurs the most during normal brain development through adulthood but can also occur in the event of a brain injury (Chudler, 2019). Although the brain is mostly influenced by genetics, the characteristics of a person's environment also affect the plasticity of the brain, according to Chudler. Plasticity is important to consider when researching the effect of different memorization strategies because children's brains have higher plasticity, so their minds are malleable and can be heavily influenced by the environment around them.

Therefore, a variety of potential strategies are revealed that must be considered when applying different memorization procedures to the abilities of children in grades four through five, also referred to as upper elementary schools. The purpose of studying such procedures would be to gain a better understanding of how children best commit information to memory. This study will identify if there is a specific style of memorization tactic that is more effective, and if so, which. Considering various memorization procedures and children also holds value because it will provide insight for teachers, parents, pediatricians, and others in the pediatric field. Providing more information on the topic will help teachers or doctors determine the most effective methods for educating children on a variety of materials.

To gain a full comprehension of the potential memorization procedures that may benefit childhood education, a variety of articles must be examined. For instance, M. B. Hargis, S. McGillivray \& A. D. Castel (2018) conducted a study on 264 undergraduate college students in which students were given two multiple-choice questions on details of the cover of a book they had been using throughout the year of one of their courses. One of the questions asked about the image on the cover of the textbook, whereas the other questioned the name of the author of the textbook (Hargis, McGillivray, $\&$ Castel, 2018). Hargis et al. found that only 39.16\% of students selected the correct answer for the first question, whereas $79.84 \%$ correctly answered the second question. The data found correlates to different memorization procedures and children in elementary schools because it supports the notion that recognition memory may be more accurate for short phrases, such as names, but less reliable in regards to remembering complex cover art as in this experiment.

In addition to memorizing short phrases and certain images, methods from India and Pakistan are often used for memorizing the Qur'an, the sacred text of Islam composed of 77,500 words. Dzulkifli and Solihu (2018) examine the Indian Deobandi method and Pakistan Panipat method, which are each divided into numerous stages. These Eastern strategies include five stages of Deobandi and seven stages of Panipat and often consist of long hours of repetition every day. To memorize the Qur'an, memorization is often done through individual and group studying, learning one or two pages each day (Dzulkifli \& Solihu, 2018). Indian and Pakistan methods of memorization are extremely important because Muslims have successfully used these techniques for centuries, but they are not much different than those found in Ancient Greece or those used today. Both articles must be considered when examining the differences among memorization procedures in upper elementary students because they reveal that recognition memory is effective in learning short phrases and long texts, although less effective in regards to complex images.

The effect of a memorization device on a child's ability to recall information greatly depends on their working memory. Magimairaj and Montgomery (2013) define working memory as a "limited-capacity system that functions to encode, store, and retrieve information being processed in any cognitive task." E. Nicolaou et al. (2017) demonstrate the significance of working memory by conducting a study on whether a low verbal and visuospatial working memory in first graders negatively impacted their working memory in third grade. The method described by Nicolaou et al. included 1,802 children in the first grade who completed both a verbal and a visuospatial working memory screening measure to single out those who have a lower working memory (E. Nicolaou et al., 2017). Two years later, 1,070 of the original students were reassessed, amounting to only 59\% of the original participants. (E. Nicolaou et al., 2017). Nicolaou et al. found that individual development of working memory varied widely among participants, although those classified as having low working memory in Grade 1 showed much larger increases than others who had "not low" working memory (E. Nicolaou et al., 2017). These results show that working memory is extremely adaptive, which is likely connected to the aforementioned neuroplasticity because children's brains are constantly changing during the developmental stage of their lives. 
Studying memorization is also beneficial for pediatricians who often interact with children. A study conducted by Principe G.F. et al. (2000) examines long-term retention of the details of a physical examination for children between three and five years old. Their method divided the three- and five-year-old participants into four groups, with three of the groups receiving a programmed experience and the fourth serving as a control. The researchers found that at an interview given 12 weeks after their original examination, the children who had participated in the programmed experiences showed better recall than the control group. The focus of this article is significant because pediatricians provide patients with an abundance of information about their health. If there are strategies that can be used to improve long-term recollection, young patients may eventually gain a better understanding of the information received during a physical examination.

Within the currently available research surrounding which memorization devices are effective for children, there is still an abundance of research to be conducted. Although there is research that compares visual, auditory, and kinesthetic strategies for memorization, there are very few studies that focus on the idea of how children memorize information. Delogu and Lilla (2017) discussed audio, visual, and verbal tactics for memorization and provided a plausible and replicable method. Their study provided subjects with stimulus material that consisted of 30 written words, 30 spoken words, 30 pictures, and 30 environmental sounds, which allowed the researchers to focus on object location memory. Participants were asked to match the material together, such as a picture of a bee with the written word "bee" and the environmental sound a bee makes, a buzzing sound. Delogu and Lilla utilize this method to focus on auditory, visual, and verbal memorization and how it is associated with object location memory. Although their article uses a plausible and reliable method, it does not focus on kinesthetic memorization, which is important because kinesthesia relies on bodily movements and tensions that provide stimulation ("Kinesthesia," n.d.). The article also focuses on how college students memorize information, which may be different than how children perform such a task because their brains are less developed. As a result, researching visual, auditory, and kinesthetic memorization procedures is important to better understand upper elementary students from a wide variety of classrooms. In addition, researchers have already studied visual and verbal memorization and comprehension in children. Reese (1975) provides such knowledge, focusing on preschool children, and how they retain information over time. However, the lack of knowledge surrounding kinesthetic memorization is still evident, although this study does provide insight into the long-term memory instead of the short term. Likewise, L. Piccardi et al. (2014) found that visual memory is the most developed working memory in children in elementary schools. It is evident, then, that visual memory is thoroughly researched in children in upper elementary schools, but that there is also a need for kinesthetic memory to be studied. Such complexities raise the question: Do students in upper elementary schools memorize a short text most efficiently through visual, auditory, or kinesthetic devices?

\section{Method}

This study implemented a mixed experimental method. Participants were randomly sampled into three separate groups, each of which attempted to memorize a poem as thoroughly as possible in 15 minutes. The poem, "My Dog Likes to Dig," is a 151-word children's poem written by Kenn Nesbitt. All three groups were given a specific device focusing on sight through a visual device, hearing through an auditory device, or touch through a kinesthetic activity, which they were given 15 minutes to interact with. The visual device can be accessed in Appendix A, the provided audio clip in Appendix B, and the kinesthetic activity in Appendix C. This amount of time was allotted so the visual and auditory groups could read or listen to their device at least seven times, given that participants took 30-second breaks in between, also allowing the visual group to make written notes. Furthermore, 15 minutes were allowed so the kinesthetic groups were able to properly conduct the activity described below.

The visual device included a printout of the poem, in which students were instructed to read through the poem and attempt to memorize it. They were allowed to read the poem as many times as they wanted and in any sequence of stanzas. The children in this group were allowed to write on the poem to visualize the poem and develop mnemonic devices or other visual aids, but they were not allowed to speak the words of the poem at all. This decision 
made it easier for the young participants to highlight or emphasize words that they thought were important to remember without deviating from the visual aspect of their activity.

The children in the group with the hearing device were given a recording of a female voice speaking the poem aloud, which they listened to as a whole group through the speakers of a MacBook Air. The audio recording is about 60 seconds long, so participants listened to the poem once all the way through at the beginning of their 15 minutes before listening to it again, repeating this step as many times as desired. Students in this group were not allowed any visual aid and could not write anything down about the poem until the assessment. This decision was an attempt to ensure that the members of this group only interacted with the poem audibly until the assessment.

The final group of participants was divided into smaller groups of 6-7 and given a printout of the poem. However, instead of reading it through to commit it to memory, each small group was given 15 minutes to come up with motions to associate with the keywords or phrases from the poem. The motions could be done with any part of their bodies, consisting of mostly arm and leg motions. These students were not allowed to write on the copy of the poem, although they had to be able to verbally communicate together and read the poem to develop their motions. Although students in this group were also given a visual aid, the primary purpose of this activity was to focus on tactile movements and memorization. The visual aid was only provided for the participants to have access to the poem they were developing motions for.

After all of the participants were given 15 minutes to work with their strategy and memorize the poem as best as possible, they were given a 15-question quiz on the poem. The quiz was a direct copy of the poem "My Dog Likes to Dig," only 15 keywords and phrases from the poems were replaced with blank spaces for the students to fill in, which is accessible in Appendix D. No list of potential phrases was provided for the students, and they were not allowed to work together during the quiz, which ensures that participants relied solely on their memory to complete the assessment. Students were given 15 minutes to complete the quiz before assessments were collected to allow all students enough time to write and review their answers.

To determine the sample size of the study, seven teachers from a local elementary school were contacted, all of whom taught fourth through fifth grade. Obtaining participants from a local elementary school satisfies the demographic I aimed to gather because the study focused on ideal memorization devices for children in upper elementary school. To ensure that the samples of each grade had a normal distribution, the ideal number of 15 males and 15 females from both grades was set, resulting in 60 students overall. This goal was set because it would amount to 30 participants per grade and 30 persons per gender so the distributions would align with a normal curve.

Within each class, each participant was assigned a number from one through three. This was done by obtaining a list of all students and using a random name generator. The first name drawn was assigned a one, the second and third names were assigned two and three accordingly, and the process was repeated for the rest of the students, proceeding with the fourth name drawn. By randomly assigning these numbers, it ensured that there was no bias in assigning participants to a specific device. This decision also ensured a similar number of participants in each group. Participants who were given a "one" received the visual device, those who were given a "two" received the listening device, and those who were given a "three" received the kinesthetic activity. Randomly sampling students into three groups to study different devices ensures that the groups were unbiased in the type of device they were given. The individual score of each participant's final assessment was the focal point of data collection. However, I also asked each participant to record their age, their gender, and the device they were given to conduct further analyses on demographics.

\section{Results}

Before the data collected can be analyzed and tested, the results must be presented. Of the seven teachers who were contacted, two fourth grade teachers and one fifth grade teacher gave their students the option to participate in this study. One student did not indicate which activity they participated in, so their response was not included in the data. Excluding this student, a total of 38 fourth graders and 18 fifth graders chose to participate and fill out the required 
assent and parent permission forms. The number of fourth-grade students exceeds the ideal number of 30 participants necessary to ensure a normal curve. Despite this, the amount of fifth graders does not exceed the ideal number. Subsequently, the goal of 60 students was not met. Likewise, the study obtained 25 male and 31 female participants, so only the females exceeded the ideal goal of 30 students. All 56 participants were between the age of 9 and 11 .

After examining the individual scores of the participants, it was found that the average score across all participants was 7.25 with a standard deviation of 3.10. Four students scored a 1 out of 15, two of whom were in fourth grade and two of whom in fifth grade. One fourth grader was a female in the kinesthetic group, while the other was a male in the visual group. Similarly, both fifth graders were males in the auditory group. In contrast, only one student earned a score of 15 out of 15 , and they were a fourth-grade female who completed the visual task.

15

Male

Female

10

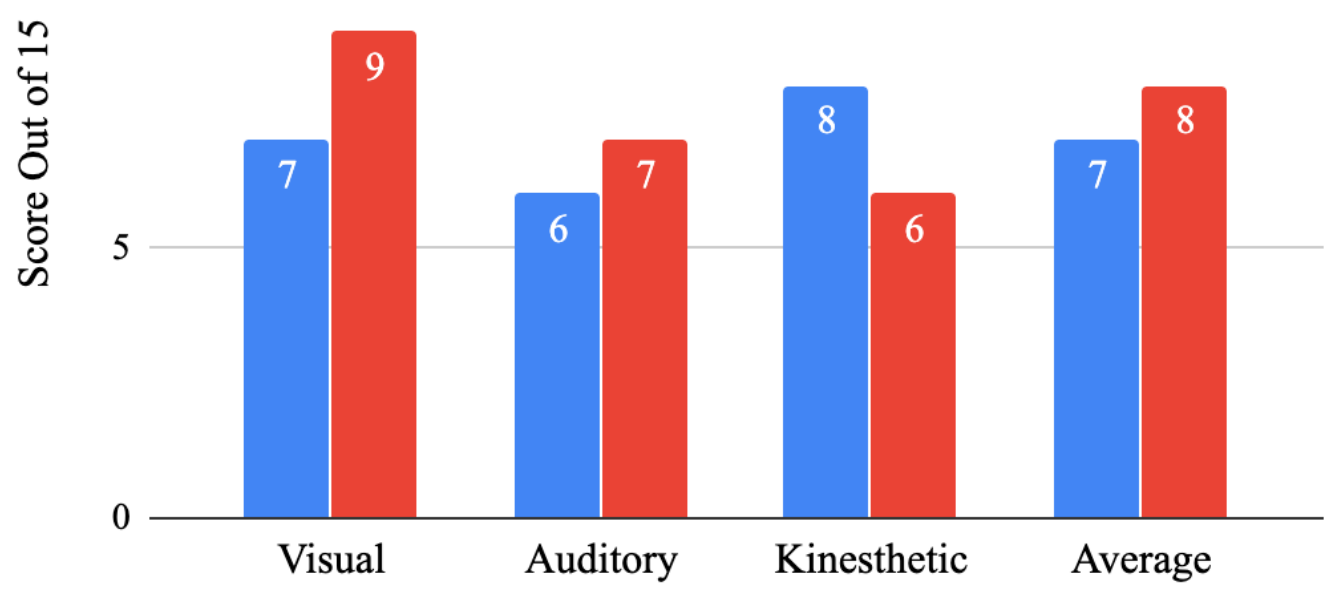

Figure 1. Average Scores Based on Gender and Device

Table 1. Average Scores Based on Grade

\begin{tabular}{|c|c|c|c|c|}
\hline & Visual & Auditory & Kinesthetic & Grade Average \\
\hline 4th Grade & 7.7 & 6.9 & 7.1 & 7.2 \\
\hline 5th Grade & 9.3 & 6.4 & 6.0 & 7.3 \\
\hline Device Average & 8.5 & 6.7 & 6.5 & \\
\hline
\end{tabular}

To determine if the scores produced by the students significantly depended on demographics, assessment scores were sorted both by gender and grade. The results for gender show that there appears to be very little difference across three devices, as shown in Figure 1. Although the differences are minor, the figure does show that female participants scored higher not only overall but also with reading and listening devices, whereas male participants scored higher with the kinesthetic device. In addition, the results for each grade show that the visual device yielded higher scores for both groups of participants, as shown in Table 1. However, the table shows that the visual groups also had the widest mean difference of 1.6, with fifth graders receiving the highest average score of 9.3 points out of 
15. In contrast, the auditory group had the smallest mean difference of 0.5 and yielded the lowest score for fourth graders. Despite these differences, the table shows that average differences between the fourth and fifth-grade scores are minimal, with a mean difference of only 0.1 .

\section{Analysis}

To conduct a statistical analysis of this data, a one-tailed t-test was conducted. This t-test was conducted using an online statistical calculator available on "SocialScienceStatistics.com." A t-test analyzing the mean difference was necessary for a comparison of the grade averages found in Table 1 to determine if the performance of one grade influenced the scores produced by each device. With a P-value of 0.48 , the test was unable to show that the fifth-grade participants yielded higher scores than fourth-grade participants at a significance level of 0.10 . Another set of t-tests were conducted using the aforementioned calculator website to determine if the reading average score is significantly higher than the audio or kinesthetic averages. To accurately analyze the data, three t-tests were conducted. The first ttest compared visual mean to audio mean, the second t-test compared auditory and kinesthetic means, and the final ttest compared the visual and kinesthetic averages. This was necessary due to the presence of three means as opposed to two, so more tests were conducted to analyze the data efficiently. The calculations found that the visual average score was significantly higher than both the audio and kinesthetic average scores with a significance level of 0.10 with P-values of 0.08 against auditory and 0.09 against kinesthetic. Despite these results, it should be noted that the sample size of this experiment included 56 participants, which is a small testing group. However, the results of this experiment still show with statistical significance that visual devices are more beneficial than auditory or kinesthetic devices when upper elementary school children are memorizing a short poem.

A variety of the implications found in the results of this study apply to elementary school classrooms and other educational environments. Visual memorization procedures were found to yield a higher mean score than other memorization procedures used in this study. As mentioned before, pediatricians will greatly benefit from gaining knowledge on how adequately children retain information because they can determine how best to communicate with their patients. This can improve communication between a doctor and a patient or their parent/guardian during physical examinations or other check-ups because they can determine what their patients are most likely to remember, although the study conducted in this paper was not focused on long-term memory. The results of this paper are also not allowed to be extended to patients outside of 9 to 12 years old. Teachers at an upper elementary school level can utilize the results of this study to refine their study plans to best suit their students, especially when involving a visual text. For instance, they can provide their students with mnemonic devices that can assist them in retaining visual memory and gain a better understanding of the capabilities of their students.

However, the scope of this experiment is limited by a few factors. A sample size of only 56 participants yields a normally distributed curve, but it prevents the results from having a significant impact. The participants in this study included an established balance between genders, but the availability of participants influenced the demographic and the sample size of the experiment. In addition, the study described in this paper only focuses on memorizing a short poem and can therefore not be applied to memorizing other types of material or retaining such information for an extended period. Another limiting factor that applies to the results is that all participants were selected from one elementary school in Northeast Ohio, so applying the conclusions of this experiment to other regions would be inappropriate due to variances that would be unaccounted for. It would also be inappropriate to apply the results of this study to other age groups as it focused on children ages 9-12. Although an attempt to reduce the occurrence of confounding factors was made when handing participants instructions for their devices and the assessment, there is a possibility that some confounding factors may have occurred regardless, such as collaboration among participants when not allowed. 


\section{Conclusion}

The results and analysis of this study show that participants did score significantly higher when using a visual device to memorize a poem. This suggests that when attempting to memorize a textual assignment, including poems, articles, and other short excerpts, visual devices may be more effective than other devices. However, these results do not apply to memorize other types of assignments, such as audio or video recordings because the study only focused on memorizing text with different devices. The study also specifically examined how children perform with memorizing information and is therefore not applicable to other age groups due to brain plasticity and constant development. Furthermore, participants took the assessment immediately following the time spent with their given device, so it would be inappropriate to apply these results to the long-term memorization of this content. The results also found that there was no significant difference in the performance of participants depending on grade, which supports the notion that children in similar age groups develop similar skills for retaining text-based content. This suggests that visual memorization procedures are more effective than auditory or kinesthetic procedures when assigning a child to commit a short text to memory for a limited period. However, these results are derived from a small sample size from a specific location since the set sample goal was not met from both grades or both genders and therefore may be limited by these factors. Although there were enough fourth graders and enough females to establish a normal curve for these samples, there were not enough male or fifth-grade participants to establish a normal curve, which may cause errors in the findings.

However, the results of this study add to the limited available research that applies to how children memorize information. This can benefit teachers who work with children in upper elementary school and aim to memorize visual texts. These teachers can provide their students with various visual memorization devices and correlating mnemonic devices to assist their memorization methods. Mnemonic devices have been used for thousands of years and are beneficial when used alongside visual devices because they provide more stimulation. Additionally, when pediatricians meet with their patients, they can have a more thorough understanding of what younger patients will retain and can improve upon the strategies they use when informing patients of their health. Specifically, providing visual devices on a computer, poster, or sheet of paper may greatly benefit the patient and their parent or guardian. The results of this study can only apply to professionals who work alongside children between the ages of 9 and 12 years old and, until numerous studies are conducted that support these findings, cannot be applied to populations outside of Northeast Ohio. It must be noted that this is only a preliminary study using different methods for memorizing information. The research shows that there is a relationship between a higher mean score and the visual device, but more substantial information is necessary before thorough conclusions can be developed. Future researchers can build upon these results by examining how children memorize other forms of content, including but not limited to audio or kinesthetic assignments. These examinations may also explore using numerous strategies in combination instead of one in isolation. Others interested in this content can also focus future studies on memorizing long passages of text or retaining a short text for an extended period. Memorization is essential for learning information, so it is important to understand what types of devices have the most beneficial impact, especially in education.

\section{Acknowledgments}

I would like to thank Ms. Patricia Talarczyk for helping me with this project.

\section{References}

Chudler, E.H. (2019). Brain plasticity: What is it? Learning and memory. https://faculty.washington.edu/chudler/plast.html 
Delogu, F., \& Lilla, C. C. (2017). Do you remember where sounds, pictures and words came from? The role of the stimulus format in object location memory. Memory, 25(10),13401346.https://doi.org/10.1080/09658211.2017.1300668

Dzulkifli, M. A., \& Solihu, A. K. H. (2018). Methods of Qur'ānic Memorisation (Ḥifž): Implications for Learning Performance. Intellectual Discourse, 26(2), 931-947. Retrieved from http://search.ebscohost.com/login.aspx?direct $=$ true $\& \mathrm{db}=\mathrm{a} 9 \mathrm{~h} \& \mathrm{AN}=133745932 \&$ site=ehost-live

Hargis, M. B., McGillivray, S., \& Castel, A. D. (2018). Memory for Textbook Covers: When and Why We Remember a Book by Its Cover. Applied Cognitive Psychology, 32(1), 39-46.

https://doi.org/10.1002/acp.3375

Kinesthesia. (n.d.). In Merriam-Webster.com Dictionary. Retrieved from https://www.merriam-webster.com/dictionary/kinesthesia

Magimairaj, B. M., \& Montgomery, J. W. (2013). Examining the Relative Contribution of Memory Updating, Attention Focus Switching, and Sustained Attention to Children's Verbal Working Memory Span. Child Development Research, 1-12. https://doi.org/10.1155/2013/763808

Nesbitt, K. (2019). My Dog Likes to Dig. Retrieved from https://www.poetry4kids.com/poems/my-dog-likes-to-dig/

Nicolaou, E., Quach, J., Lum, J., Roberts, G., Spencer-Smith, M., Gathercole, S., .. Wake, M. (2017). Changes in verbal and visuospatial working memory from Grade 1 to Grade 3 of primary school: Population longitudinal study. Child: Care, Health and Development, 44(3), 392-400. doi: 10.1111/cch.12543

Piccardi, L., Leonzi, M., D’Amico, S., Marano, A., \& Guariglia, C. (2014). Development of navigational working memory: Evidence from 6- to 10-year-old children. British Journal of Developmental Psychology, 32(2), $205-217$. https://doi.org/10.1111/bjdp.12036

Principe, G. F., Ornstein, P. A., Baker-Ward, L., \& Gordon, B. N. (2000). The effects of intervening experiences on children's memory for a physical examination. Applied Cognitive Psychology, 14(1), 59-80.

https://doi.org/10.1002/(SICI)1099-0720(200001)14:1<59::AID-ACP637>3.0.CO;2-4

Reese, H. W. (1975). Verbal Effects in Children's Visual Recognition Memory. Child Development, 46(2), 400407. https://doi.org/10.2307/1128134

Sarma, G. P. (2015). Reconsidering Written Language. Interdisciplinary Description of Complex Systems, 13(3), 397-404. https://doi.org/10.7906/indecs.13.3.5

Social Science Statistics. (n.d.). Retrieved March 23, 2020, from http://www.socscistatistics.com/Default.aspx

Vatri, A. (2015). Ancient Greek Writing for Memory. Mnemosyne, 68(5), 750-773.

https://doi.org/10.1163/1568525X-12341688 